\title{
Incidental Finding of Mesothelioma after a Motor Vehicle Accident
}

Tariq Khurram, Lala Roshan, Khousakoun Somphanh, Farhangi Arezo, Rana Fauzia

University of Florida, Department of Internal Medicine, Division of Hematology and Medical Oncology, USA

*Corresponding author: Khurram Bilal Tariq, MD, University of Florida, Department of Internal Medicine, 653 West 8th Street, Box L 18, Jacksonville, FL 32209, USA, Tel: 904-244-3093; E-mail: Khurram.Tariq@jax.ufl.edu

Received: June 27, 2014; Accepted: July 02, 2014; Published: July 03, 2014

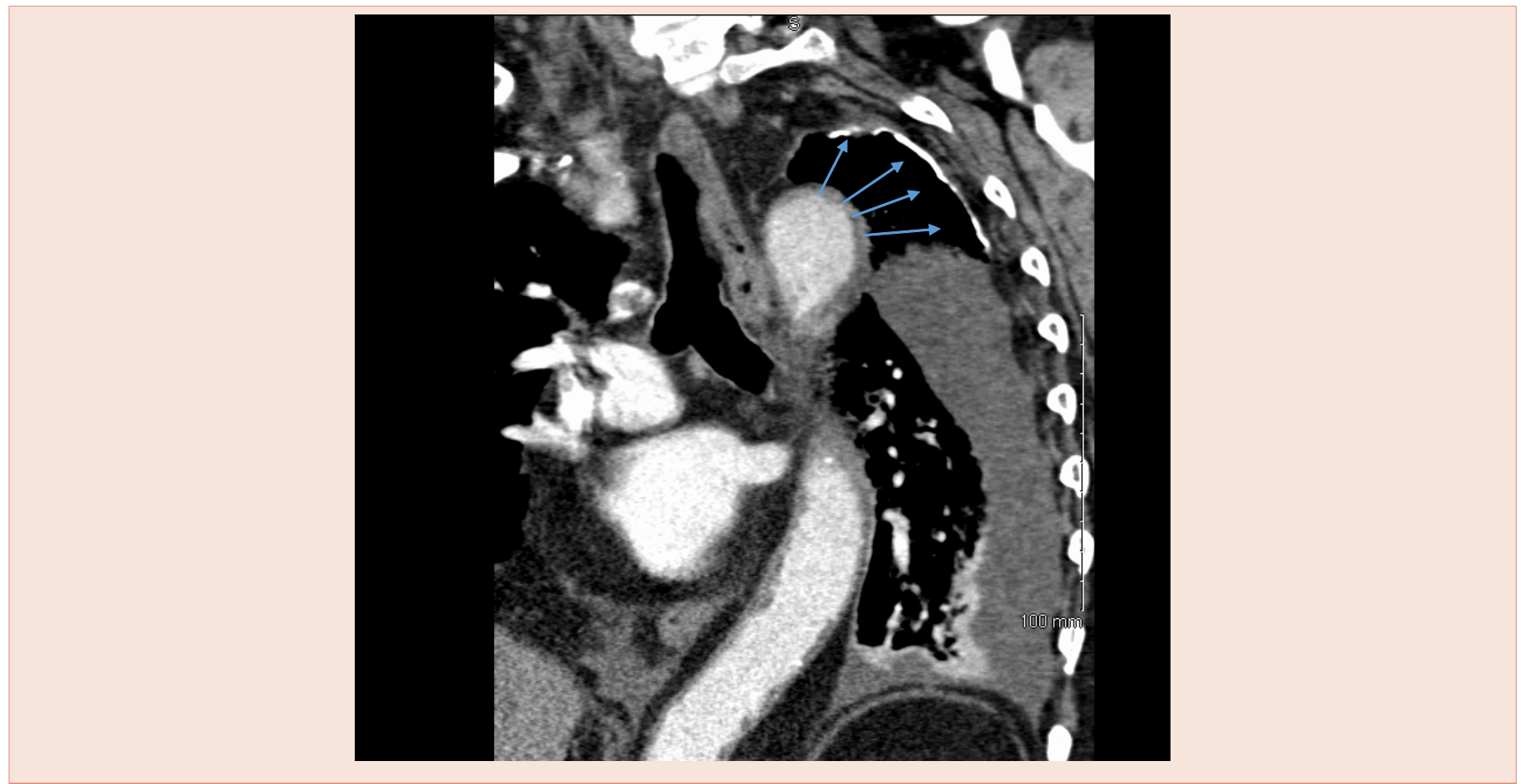

CTA Chest: The blue arrows point to the pleural calcification seen in this patient with asbestos exposure.

Patient is a 76 year old male who suffered from severe asbestos exposure while working for the plumbing industry. He Presented to the emergency department after a motor vehicle accident and on workup was incidentally found to have bilateral pleural effusions and calcification of the pleura in the left lung consistent with Mesothelioma. 